Internist 2005 · 46:1096-1104 DOI 10.1007/s00108-005-1457-y

Online publiziert: 1. Juli 2005

(c) Springer Medizin Verlag 2005

Schwerpunktherausgeber

M. Wehling, Mannheim

\author{
J. M. Gschossmann ${ }^{1}$ - G. Holtmann ${ }^{2}$. P. Netzer ${ }^{1} \cdot$ M. Essig ${ }^{1}$ B. M. Balsiger ${ }^{1}$ \\ U. Scheurer ${ }^{1}$ \\ ${ }^{1}$ Klinik für Gastroenterologie, Inselspital/Universität Bern, Schweiz \\ ${ }^{2}$ Department of Gastroenterology, Hepatology \& General Medicine, \\ Royal Adelaide Hospital - University of Adelaide, Australien
}

\title{
Abdominaler Schmerz
}

\begin{abstract}
A ten primär als „Bauchschmerzen“ angegeben, weisen eine hohe Prävalenz auf. So berichteten 3 von 4 gesunden Studenten in einer Befragung von abdominalen Schmerzen [15]. In einer anderen epidemiologischen Studie von Heading [10] gab die Hälfte der befragten Bevölkerung an, gelegentlich unter abdominalen Schmerzen $\mathrm{zu}$ leiden. Insgesamt stellen abdominale Schmerzen neben kardialen und pulmonalen Beschwerden die häufigsten Symptomkomplexe dar, mit denen der in der Primärversorgung tätige Arzt konfrontiert wird. Angesichts einer Vielzahl unterschiedlicher Differenzialdiagnosen abdominaler Schmerzen ist ein systematisches Vorgehen in Diagnostik und Therapie notwendig, um die zu ergreifenden Maßnahmen an den jeweiligen Patienten adaptieren zu können. Ziel der vorliegenden Uebersicht ist es, das breite Themenspektrum des „abdominalen Schmerzes“ zu strukturieren und Ansatzpunkte zur Erstellung eines persönlichen Logarithmus bei Vorstellung eines Patienten mit derartigen Beschwerden zur Hand zu geben.
\end{abstract}

\section{Abdominale Schmerztypen}

Vor der klinischen Untersuchung eines Patienten mit abdominalen Schmerzen ist es von entscheidender Bedeutung, dass sich der Untersucher vergegenwärtigt, dass es nicht den für alle Situationen klassischen Abdominalschmerz gibt. Im Gegenteil lässt sich der abdominale Schmerz in verschiedene, sich voneinander typischerwei- se abgrenzende Schmerzarten unterteilen (• Abb. 1).

Als erster Subtyp des abdominalen Schmerzes ist der somatische Schmerz zu erwähnen. Dieser entsteht v. a. durch Irritation der Abdominalwand bzw. des Peritoneum parietale [18]. Vom Patienten wird dieser Schmerz als genau lokalisierbar und intensiv beschrieben. Die Schmerzleitung erfolgt hierbei über schnelle A-Nervenfasern. Der somatische Schmerz erfährt eine Verstärkung durch Lageveränderungen, sodass eine reflektorische Schonhaltung der Abdominalmuskulatur erfolgt.

\section{( Es gibt nicht den für alle Situationen klassischen Abdominalschmerz}

Im Gegensatz dazu steht der viszerale Schmerz, welcher seinen Ursprung in den Eingeweideorganen bzw. im Peritoneum viscerale hat $[16,19]$. Der viszerale Schmerz wird vom Patienten als schwer lokalisierbarer, dumpfer oder brennender Schmerz empfunden [8]. An der Schmerzleitung sind langsame $\mathrm{C}$-Nervenfasern beteiligt [3]. Viszerale Schmerzen treten oft in Verbindung mit vegetativen Symptomen wie Nausea, Vomitus und Kreislaufalterationen auf.

Der übertragene Schmerz ist durch afferente Koinnervation spinothalamischer Bahnen durch viszerale und kutane Nervenfasern bedingt. Das klinische Korrelat sind schmerzhafte Sensationen in den entsprechenden Dermatomen (Head-Zonen) oder Myotomen. Klassische Beispie- le eines übertragenen Schmerzes sind die schmerzende linke Schulter durch Milzläsionen (Saegesser-Zeichen) sowie ein Schmerzempfinden in der rechten Schulter durch pathologische Alterationen im Bereich der Gallengänge [27].

Nicht im direkten Zusammenhang mit abdominellen Erkrankungen stehend, aber trotzdem als abdominale Schmerzen vom Patienten angegeben, können der projizierte Schmerz sowie der psychogene Schmerz sein. Der projizierte Schmerz resultiert aus einer Irritation von peripheren Nervenbahnen, Nervenwurzeln oder Plexusläsionen, welche durch Neoplasien, Inflammationen oder Diskusprolaps bedingt sein können. Wahrgenommen werden diese Schmerzen vom Patienten im peripheren Ausbreitungsgebiet der betroffenen Nerven. Dies hat zur Folge, dass eine oft zur initialen Verwirrung führende örtliche Distanz zwischen dem eigentlichen Erkrankungsort und der vom Patienten empfundenen Schmerzlokalisation vorliegen kann. So können Bandscheibendegenerationen, welche zu einer Irritation der distal gelegenen thorakalen Spinalnerven führen, mit Unterbauchschmerzen assoziiert sein. Von Bedeutung für die Diagnose ist hierbei auch die Tatsache, dass die diesbezüglichen Schmerzen lageabhängig unterschiedlich und durch Alterationen des intraabdominalen Druckes variieren können.

Beim psychogenen Schmerz kann es sich zum einen um eine vorsätzliche Schmerzvortäuschung handeln. Weitverbreitet sind hierbei hysteriforme Reaktionen mit sekundärem Krankheitsge- 


\section{Hier steht eine Anzeige.}

黛 Springer 

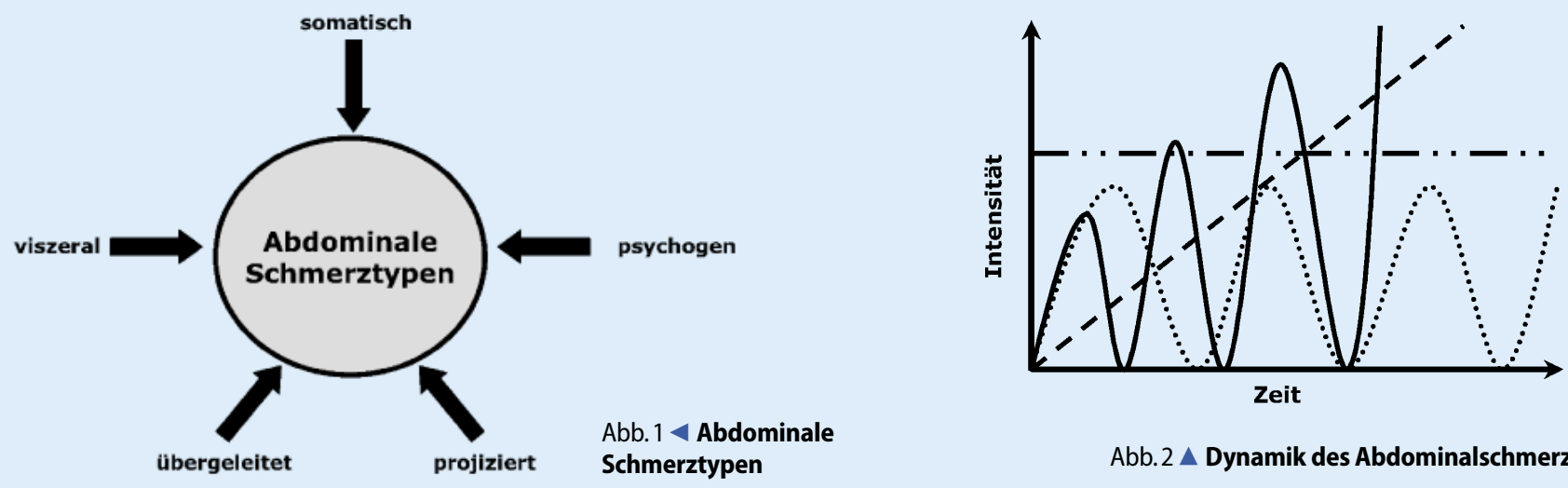

Abb. $1<$ Abdom
Schmerztypen

Abb. $2 \Delta$ Dynamik des Abdominalschmerzes
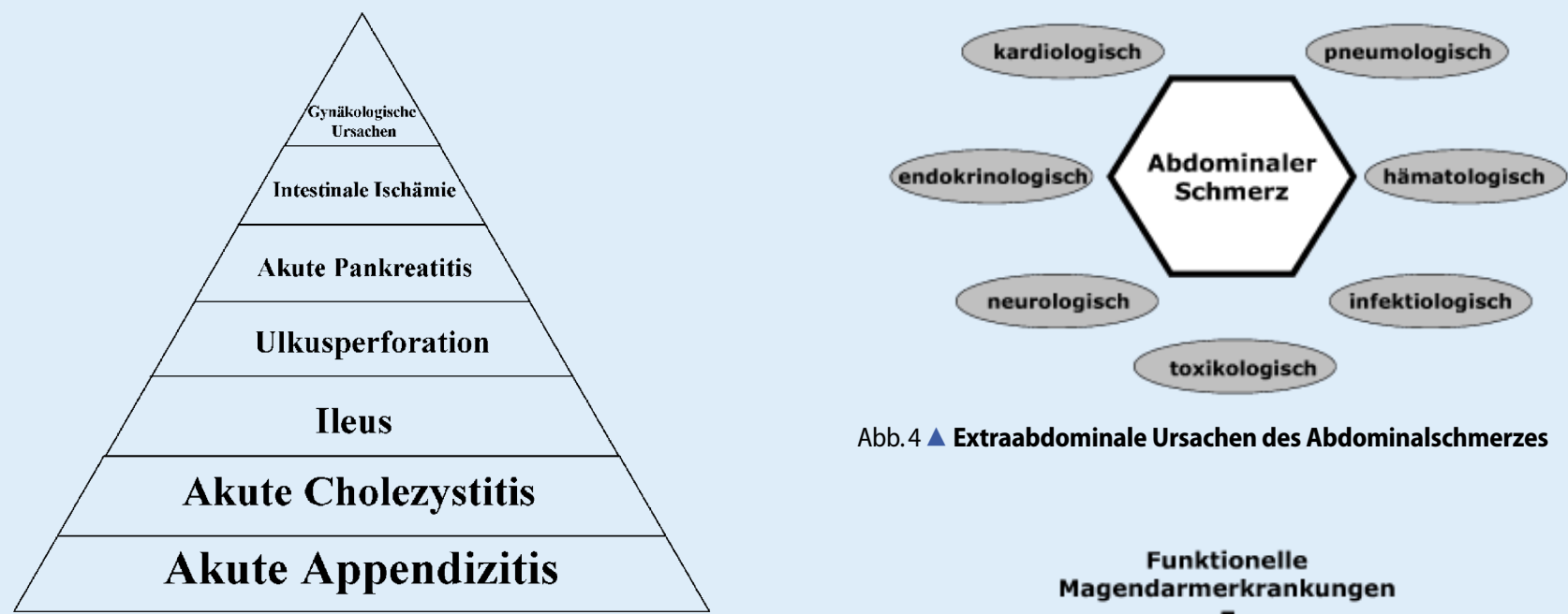

Abb. 4 Extraabdominale Ursachen des Abdominalschmerzes

Abb. $3 \Delta$ Ursachen des akuten Abdomens

Abb. 5 Ursachen chronischer Abdominalschmerzen

winn vor dem Hintergrund eines Begehrens (z. B. Rentenanspruch). Zum anderen sind jedoch auch psychogene Schmerzen im Zusammenhang mit psychischen Alterationen wie schweren Depressionen, Angstzuständen und aggressiven Verhaltensmustern bekannt.

\section{Diagnose abdominaler Schmerzen}

Für eine korrekte Beurteilung der abdominalen Schmerzen und Festlegung der daraus resultierenden therapeutischen $\mathrm{Maß}$ nahmen sind eine präzise Anamnese und körperliche Untersuchung von entscheidender Bedeutung [2].

Bei der Anamneseerhebung gilt es die genaue Lokalisation (lokalisiert oder wechselnd) zu erfragen. Mittels der Lokalisation der Schmerzen kann das Spektrum der möglichen Differenzialdiagnosen bereits eingegrenzt werden ([30]; - Tabelle 1). Ebenfalls von Bedeutung für die Einengung möglicher Schmerzursachen sind Angaben zum zeitlichen Verlauf (akut versus chronisch, progredient versus stabil) sowie zum Schmerzcharakter (anfallsartig versus permanent, dumpf versus stechend; $\bullet$ Abb. 2). Wichtig ist auch die Familien- und Medikamentenanamnese (z. B. NSAR). Auch kön- nen Informationen zu mildernden bzw. die Schmerzen steigernden Einflussfaktoren (z. B. Zusammenhang mit Menstruation) und möglichen Begleitsymptomen wie Nausea/Vomitus und Stuhlveränderungen oder Ikterus essenziell sein.

Bei der klinischen Untersuchung eines Patienten mit abdominalen Schmerzen können im Rahmen der Inspektion durch Registrierung von Haut- und Konturveränderungen bereits erste wertvolle Hinweise auf die zugrunde liegende Erkrankung des Patienten gewonnen werden. Die Palpation und Perkussion dient dazu, pathologische Resistenzen sowie Organomegalien nachzuweisen. Das Vorlie- 
gen einer lokalen Défense bzw. eines peritonitischen Abdomens liefert neben klinischen Hinweisen wertvolle Informationen zur Dringlichkeit der weiteren Diagnostik und Therapie. Bei der Auskultation des Abdomens sollte das Augenmerk v. a. den Darmgeräuschen gelten.

Obligater Bestandteil einer Untersuchung des Abdomens ist auch die Rektaluntersuchung, welche ebenfalls aus Inspektion und Palpation besteht. Nach einer visuellen Beurteilung der Perianalregion ist mittels digitaler rektaler Untersuchung eine Evaluation der Sphinkterfunktion sowie eine Beurteilung der Oberfläche von Analkanal und Rektumampulle möglich. Aus der Palpation der Rektumampulle resultieren ferner wichtige Hinweise zur Oberflächenstruktur und Beschaffenheit perirektaler Strukturen sowie Stuhlkonsistenz und mögliche Blutbeimengungen.

Die zur Abklärung abdominaler Schmerzen indizierten Laboruntersuchungen sind in grundlegende und weiterführende Bestimmungen zu unterteilen. Die diesbezügliche Basisdiagnostik (• Tabelle 2) umfasst Blutbild und Entzündungsparameter, Leber-, Cholestase- und Pankreaswerte. Daneben sollten Serumelektrolyte und Blutglukose gemessen und Urinstatus bestimmt werden. Art und Umfang der weiterführenden Labordiagnostik ist vom Ergebnis der Anamneseerhebung und der körperlichen Untersuchung abhängig.

Eine entscheidende Ergänzung für die Diagnosefindung bei Vorliegen abdominaler Schmerzen stellen bildgebende Verfahren dar [26]. Auch hierbei ist wiederum zwischen Basismaßnahmen und einer individuellen, der jeweiligen Situation adaptierten Eskalation der Bildgebung zu unterscheiden.

Nicht zuletzt wegen der allgemeinen Verfügbarkeit, der Schnelligkeit der Untersuchung und des nichtinvasiven Charakters bei fehlender Strahlenbelastung spielt hierbei die Sonographie eine entscheidende Rolle, welche bereits vom behandelnden Hausarzt oder dem diensthabenden Arzt auf der Aufnahmestation durchgeführt werden kann. Endoskopische Untersuchungen des oberen und unteren Gastrointestinaltraktes, aber auch Endosonographie und endoskopisch-retrograde Cholangiopankreatikographie stellen Erweiterungen der apparativen Diagnostik

Internist 2005 - 46:1096-1104

DOI 10.1007/s00108-005-1457-y

(c) Springer Medizin Verlag 2005

\section{J. M. Gschossmann · G. Holtmann · P. Netzer · M. Essig · B. M. Balsiger · U. Scheurer}

\section{Abdominaler Schmerz}

\section{Zusammenfassung}

Abdominalschmerzen können Ausdruck einer Vielzahl intra- und extraabdomineller Erkrankungen sein. Angesichts dieses breiten ätiologischen Spektrums gilt es, im Initialstadium der Diagnostik zielgerichtet vorzugehen, um ohne Zeitverzug die Ursache und damit letzlich die Dringlichkeit weitergehender Maßnahmen zu bestimmen. Ziel dieser Evaluation ist eine initiale risikostratifizierte Triagierung des Patienten. Im Gegensatz zu somatischen Ursachen abdominalen Schmerzes ist eine derartige kausale

\section{Abdominal pain}

Abstract

Abdominal pain can result from a variety of different intra- and extra-abdominal disorders. Given the wide variety of etiological triggers for this pain, the primary task during the first stage of the diagnostic workup is to determine as soon as possible the underlying cause and the degree of emergency. The aim of this evaluation is to adapt the therapeutic measures which are necessary for a causal treatment to the individual situation. Contrary to somatic caus-
Therapie bei funktionellen Erkrankungen des Gastrointestinaltraktes zum gegenwärtigen Zeitpunkt nur sehr begrenzt möglich, sodass hierbei der Fokus auf eine bedarfsund symptomadaptierte Behandlung gelegt werden soll.

\section{Schlüsselwörter}

Abdominaler Schmerz - Somatischer Schmerz • Viszeraler Schmerz .

Übertragener Schmerz · Kausale Therapie es of abdominal pain, the availability of such a causal therapy for functional bowel disorders is still very limited. Given this dilemma, the therapeutic focus of abdominal pain associated with these functional syndromes has to be placed on symptomoriented treatment.

Keywords

Abdominal pain · Somatic pain - Visceral pain - Referred pain - Causal treatment 
Tabelle 1

Korrelation Lokalisation und abdominale Ätiologie des Abdominalschmerzes

\section{Schmerzmaximum im rechten \\ Oberbauch \\ - Cholezystitis \\ - Ulcus ventriculi \\ - Ulcus duodeni \\ - Pankreatitis (Cave: gürtelförmig) \\ - Pyelonephritis \\ - Nephrolithiasis \\ - Retrozökale Appendizitis}

Schmerzmaximum im linken Oberbauch

- Ulcus ventriculi

- Pankreatitis (Cave: gürtelförmig)

- Milzinfarkt

- Pyelonephritis

- Nephrolithiasis

Schmerzmaximum im rechten

Unterbauch

- Appendizitis

- lleitis terminalis

- Kolitis im Bereich des Zökum,

Colon ascendens/Colon transversum

- Läsionen/Irritationen des Ureters

- Affektionen der Adnexe

Schmerzmaximum im linken

Unterbauch

- Kolitis im Bereich des Colon

transversum/descendens sowie

Sigma/Rektum

- Läsionen/Irritationen des Ureters

- Affektionen der Adnexe

durch den Gastroenterologen dar. Seit kurzem ist darüber hinaus mittels Kapselendoskopie eine Komplettierung der internistischen Bildgebung des Verdauungstraktes möglich geworden.

Radiologische Untersuchungsmethoden im Rahmen der Abklärung abdominaler Schmerzen sind neben (Kontrast)röntgenuntersuchungen die Computertomographie und die Magnetresonanztomographie. Bei Verdacht auf angiologische Ätiologie der abdominalen Schmerzen besteht ferner die Möglichkeit zur Angiographie.

\section{Differenzialdiagnose abdominaler Schmerzen}

Ziel der o. a. diagnostischen Schritte ist die Identifikation der Schmerzursache. Damit in Zusammenhang steht als erste

Tabelle 2

Basislaborparameter

zur Abklärung abdominaler

Schmerzen

Hämatologie
• Hämoglobin/Hämatokrit
- Leukozyten
-Thrombozyten
- Differenzialblutbild
Chemie
-CRP
-ASAT/ALAT
- $\mathrm{Y}$-GT
-AP
-Amylase/Lipase
- Glukose

Urinstatus

- Leukozyten

- Erythrozyten

-Zylinder

prioritäre Frage die Klärung der Dringlichkeit eines therapeutischen Handlungszwanges. Handelt es sich bei dem konkreten Fall um ein akutes oder eher chronisches Krankheitsbild?

\section{Akute Abdominalschmerzen}

Prinizipiell ist ein akutes abdominelles Schmerzereignis bis zum Beweis des Gegenteils als medizinische Notfallsituation einzustufen. Diese erfordert eine umgehende diagnostische Abklärung und in Abhängigkeit von dem daraus resultierenden Ergebnis eine entsprechende ursächliche Therapie. Für die Dringlichkeit eines akuten Abdominalschmerzes spricht das erstmalige Auftreten, die Schmerzdynamik sowie die Intensität des Schmerzes. Von Bedeutung sind darüber hinaus kardiovaskuläre und respiratorische Begleiterscheinungen wie Hypotonie und Tachypnoe, aber auch Allgemeinsymptome wie Fieber, Nausea und Vomitus.

Bei der körperlichen Untersuchung stellt v. a. ein Peritonismus ein Alarmsignal dar. So umfasst das klinische Bild eines akuten Abdomens neben einem massiven Abdominalschmerz die Parameter Abwehrspannung und Schock [17]. Besonders bei immunsupprimierten Patienten und Senioren können diese Alarmsymptome jedoch fehlen oder nur sehr diskret vorliegen, sodass eine deutliche Diskrepanz zwischen der Schwere der zugrunde liegenden Pathologie und dem Grad der klinischen Symptomatik vorliegt [4, 29].

Einen ersten Hinweis auf die Ätiologie der Abdominalschmerzen gibt der zeitliche Rahmen des Schmerzbeginnes. Während ein perakuter Beginn innerhalb von Sekunden klassischerweise bei einer Ruptur oder Perforation eines Hohlorganes auftritt, spricht ein akuter Schmerzbeginn innerhalb von Minuten eher für einen ischämischen Prozess oder ist Ausdruck einer Kolik. Entwickelt sich der akute Abdominalschmerz innerhalb von Stunden, ist differenzialdiagnostisch am ehesten ein inflammatorisches, neoplastisches oder obstruktives Geschehen auszuschließen.

Von zentraler Bedeutung ist die Entscheidung, ob das weitere Vorgehen operativer Natur sein muss oder ein zumindest initial konservatives Vorgehen zu vertreten ist. Hierzu ist nach initialer Basisdiagnostik eine frühzeitige interdisziplinäre Betreuung des Patienten durch den konsiliaren Beizug eines Chirurgen notwendig. Zwar ist die akute Appendizitis weiterhin die häufigste Ursache für das klinische Bild eines akuten Abdomens, doch ist die potenzielle Ätiologie dieses Krankheitsbildes vielfältig ([24]; • Abb. 3).

Für die Diagnosefindung erschwerend ist hierbei auch die Tatsache, dass neben abdominalen Ursachen auch extraabdominale Erkrankungen zu akuten Abdominalschmerzen führen können ( $\bullet$ Abb. 4). Eine weitere potenzielle Hürde auf dem Weg zur Diagnose ist ein unreflektierter Einsatz von Analgetika in der Initialphase der Beschwerden. Gefährlich ist hierbei die Schmerzlinderung ohne Therapie der zugrunde liegenden Erkrankung, sodass der wirkliche Erkrankungs- und Gefährdungsgrad des Patienten fälschlicherweise verharmlost wird. Aus diesem Grund sollte der Einsatz von Analgetika bis zur initialen Klärung der Schmerzätiologie zum Schutz des Patienten sehr restriktiv praktiziert werden. Auch kann ein unreflektierter Einsatz von Analgetika sogar kontraproduktiv hinsichtlich der Symptomintensität sein. Exemplarisch sei hier auf den Einsatz von Opiaten bei Vorliegen eines Ileus hingewiesen.

Zusammenfassend ist bei Vorliegen von akut aufgetretenen Abdominalschmerzen vorrangige Aufgabe des Erstuntersuchers, mittels eines diagnostischen Basis- 
schemas die spezifische Schmerzsituation des Patienten richtig einzuschätzen und in Abhängigkeit von der Dringlichkeit die weiteren diagnostischen und therapeutischen Schritte einzuleiten. Hierbei sollte sich der Berufsanfänger nicht scheuen, umgehend den erfahrenen Kollegen und der Internist den chirurgischen Kollegen hinzuzuziehen.

\section{Chronische Abdominalschmerzen}

Auf das Abdomen bezogene chronische Schmerzen können Ausdruck einer Vielzahl von Erkrankungen sein, welche in erster Linie gastroenterologischer Natur sind (- Abb. 5). Nicht zu vernachlässigen bei der Differenzialdiagnose sind aber auch extragastrointestinale Erkrankungen, wobei exemplarisch kardiale, aber auch pulmonologische Erkrankungen erwähnt seien [25].

Der Begriff des chronischen Abdominalschmerzes umfasst sämtliche subjektiv als unangenehm bewerteten Sensatio- nen, die auf das Abdomen bezogen werden. Eine strenge Unterscheidung zwischen unspezifischen Beschwerden, wie z. B. einem Völlegefühl nach einer Mahlzeit oder einem postprandialen Schmerz, ist insofern nicht immer möglich, und die Übergänge zwischen einfachen Symptomen und Schmerz sind fließend. Ähnlich wie der akute abdominale Schmerz kann auch der chronische Abdominalschmerz multifaktoriell ausgelöst werden.

Die chronischen Schmerzen zugrunde liegenden Mechanismen sind aktuell noch nicht abschließend verstanden. So konnte erst kürzlich demonstriert werden, dass zumindest im Rattenmodell neben neuronalen Zellen auch mikrogliale Zellen des Rückenmarks an der Entstehung und Vermittlung chronischer Schmerzen beteiligt sind [20]. Strukturelle Läsionen oder biochemische Abnormalitäten, die geeignet sind, die Schmerzen kausal zu erklären, finden sich aber nur bei einem Teil der Betroffenen. Sind eindeutige Ursachen der
Schmerzen nicht erkennbar, wird definitionsgemäß von einer funktionellen Störung ausgegangen [11, 28]. Sie zählen zu den häufigsten gastroenterologischen Erkrankungen [5].

Funktionelle Erkrankungen des Gastrointestinaltraktes können prinzipiell in sämtlichen Regionen des Verdauungstraktes auftreten. So ist das Krankheitsbild der funktionellen Dysphagie ebenso bekannt wie das Syndrom einer funktionellen fäkalen Inkontinenz. Im Bereich des Oberbauches nimmt die funktionelle Dyspepsie unter den funktionellen gastrointestinalen Erkrankungen die zentrale Stellung ein. Die diesbezüglichen Beschwerden können ein breites Spektrum abdecken (z. B. Schmerz, Völlegefühl, retrosternales Brennen, Druck im Oberbauch, frühzeitiges postprandiales Sättigungsgefühl) und isoliert oder in Kombination auftreten.

Funktionelle Beschwerden im unteren Abdomen werden unter dem Begriff des Reizdarmsyndroms subsummiert. Hier-

\section{Hier steht eine Anzeige.}

\section{包 Springer}




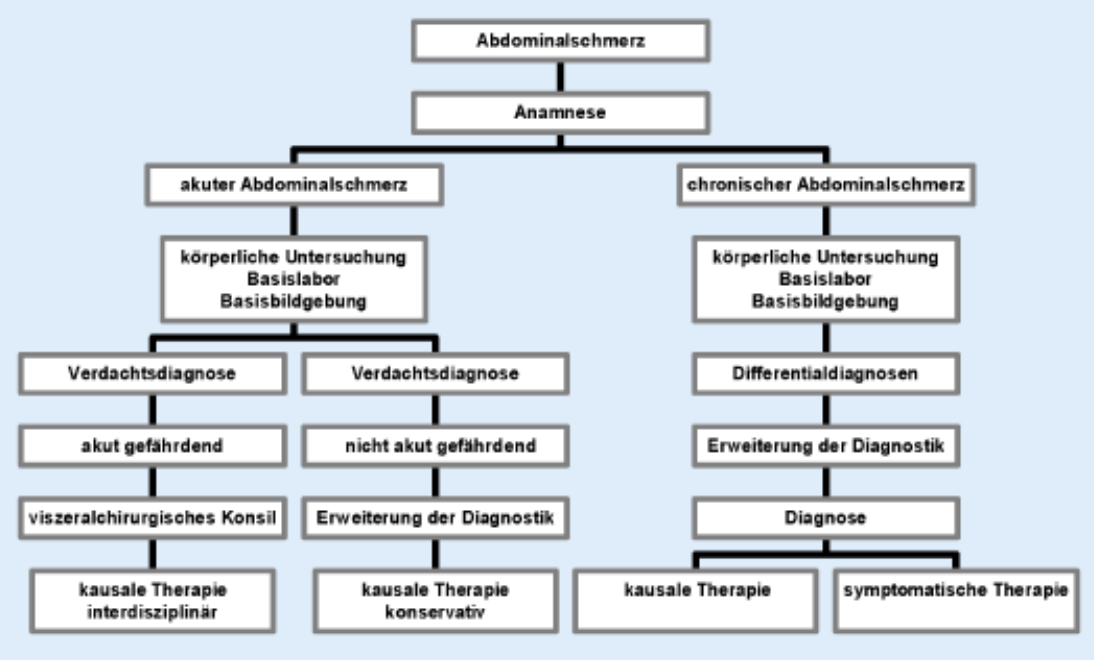

Abb. $6 \Delta$ Algorithmus zum diagnostisch-therapeutischen Vorgehen bei Abdominalschmerzen

bei berichten die Patienten neben im Unterbauch lokalisierten Schmerzen v. a. über Veränderungen der Defäkation. In einer Konsensuskonferenz über funktionelle Magen-Darm-Erkrankungen, deren Definitionen als Rom-II-Kriterien publiziert wurden, wird für die Diagnose einer funktionellen Dyspepsie oder eines Reizdarmsyndroms eine Beschwerdedauer von insgesamt 12 Wochen während der letzten 12 Monate gefordert [22].

Auch wenn aufgrund der klinischen Symptomatik und der Lokalisation der Beschwerden die Krankheitsbilder der funktionellen Dyspepsie und des Reizdarmsyndroms unterschieden werden können, ist die Überlappung dieser beiden Krankeitsbilder bemerkenswert [12]. Gleichzeitig sind die verschiedenen Symptomkomplexe im Verlauf höchst variabel. So können Patienten, die z. B. initial an einem Reizdarmsyndrom leiden, im Verlauf einen Wandel des Krankheitsbildes erfahren und später an einer funktionellen Dyspepsie oder einer Kombination beider Krankheitsbilder leiden [1]. Dies deutet an, dass den Beschwerden möglicherweise nicht eine isolierte Störung eines Organs zugrunde liegt, sondern dass übergeordnet entweder die Regulation verschiedener Organe oder die Verarbeitung der viszeralen Afferenzen gestört ist.

In den letzten Jahren konnten verschiedene Funktionsstörungen der viszeralen Nozizeption identifiziert werden, die geeignet sind, die Entstehung der Symptome bei Patienten mit funktionellen MagenDarm-Erkrankungen zu erklären [21].
Die exakten Mechanismen im Rahmen der Entstehung einer viszeralen Hyperalgesie sind bislang unbekannt, wenngleich vorstellbar ist, dass periphere Mechanismen (z. B. Entzündung der Schleimhaut) oder zentrale Faktoren (z. B. Aktivierung des Locus coeruleus oder Stress), welche eine Aktivierung der endogenen schmerzinhibierenden Systeme verhindern, eine Rolle spielen können. Den Stellenwert einer gestörten viszeralen Nozizeption in diesem Zusammenhang dokumentierte auch die klinische Beobachtung, dass nicht das Ausmaß einer aspirinbedingten Schädigung der Magenmukosa, sondern vielmehr eine fehlende Erhöhung der sensorischen Perzeptionsschwellen während einer Aspirintherapie mit der Entwicklung dyspeptischer Beschwerden assoziiert ist [13].

Aus tierexperimentellen Untersuchungen kann abgeleitet werden, dass temporäre entzündliche Schleimhautveränderungen (z. B. nach einem gastrointestinalen Infekt) anhaltend viszerale Wahrnehmungsschwellen vermindern (und eine sog. Hyperalgesie eintritt) bzw. dass Krankheitsbilder wie das sog. Reizdarmsyndrom auftreten können $[6,9]$. Dabei werden lang anhaltende Veränderungen viszeraler Wahrnehmungsschwellen nur bei einem kleinen Teil der von einer infektiösen oder entzündlichen Darmerkrankung betroffenen Patienten beobachtet [23]. Diese interindividuellen Unterschiede sind möglicherweise durch genetische Faktoren bestimmt, die bei der Ausbildung einer viszeralen $\mathrm{Hy}$ peralgesie von Bedeutung sind. So konnten
Holtmann et al. [14] erst kürzlich eine Assoziation zwischen dem G-Protein- $\beta 3$-Subtyp-825-CC-Genotyp und unerklärten dyspeptischen Beschwerden aufzeigen.

\section{Therapie abdominaler Schmerzen}

Wichtig im Hinblick auf die Therapie von Abdominalschmerzen ist die Abgrenzung organischer Ursachen von funktionellen Beschwerden. Hierbei ist zu betonen, dass die Diagnose einer funktionellen MagenDarm-Erkrankung stets eine Ausschlussdiagnose sein sollte. Von entscheidender Bedeutung neben der Durchführung unterschiedlicher Diagnoseverfahren ist bezüglich der Abgrenzung gegenüber organischen Ursachen v. a. auch die Bewertung der vom Patienten geschilderten Symptome hinsichtlich Dauer, Intensität, Progredienz und Begleitbeschwerden.

Ziel aller Therapien bei Patienten, deren abdominale Schmerzsymptomatik mit einer erkennbaren somatischen Erkrankung assoziiert ist, muss die kausale Behandlung der zugrunde liegenden Grunderkrankung sein. Hierzu wird auf die entsprechenden Lehrbücher verwiesen. Eine symptomatische Schmerztherapie sollte idealerweise nach initialer Diagnostik nur überbrückend eingesetzt werden müssen, bis die kausalen Therapiemaßnahmen wirksam werden und zu einer Schmerzreduktion führen.

\section{(7) Die Diagnose einer funktionellen Magen-Darm- Erkrankung sollte stets eine Ausschlussdiagnose sein}

Während hierbei das therapeutische Vorgehen abhängig von der im Einzelfall nachgewiesenen strukturellen Läsion oder biochemischen Abnormalität ist, muss bis dato das weitgehende Fehlen einer kausalen Behandlungsoption für Patienten mit funktionellen Magen-Darm-Erkrankungen konstatiert werden. Auch kann nur in einem Teil der Patienten mit derartigen funktionellen Beschwerden eine persistierende Beschwerdefreiheit erreicht werden. Neben Allgemeinmaßnahmen stehen verschiedene medikamentöse und psychotherapeutische Ansätze zur Verfügung. Die Wahl des jeweiligen Medikamentes soll- 
te in Abhängigkeit vom führenden Symptom erfolgen.

Eine symptomatische Schmerztherapie sollte nach einen Stufenschema aufgebaut sein. Hierbei gilt es in erster Linie, Allgemeinmaßnahmen von pharmakologischen Interventionen zu unterscheiden [7].

Hinsichtlich der Allgemeinmaßnahmen sollte das Augenmerk v. a. auf der Identifikation und Modifikation von Lebenseinflüssen gerichtet sein, welche anamnestisch im Zusammenhang mit Schmerzbeginn oder Schmerzaggravation eingeordnet werden können. Ähnliches gilt für die Nahrungsmittelanamnese. Auch hierbei ist es gelegentlich bereits hilfreich, mittels eines Ernährungstagebuches probatorisch Veränderungen der täglichen Nahrungszufuhr durchzuführen.

Führen diese Maßnahmen zu keinem Erfolg, besteht mittels einer medikamentösen Therapie die Möglichkeit, eine Schmerzlinderung direkt über eine direkte Beeinflussung viszeraler Afferenzen oder indirekt über eine Beeinflussung von gastrointestinalen Funktionen anzustreben.

Der Einsatz von nichtsteroidalen Antirheumatika als peripher wirkende Analgetika ist bei Abdominalschmerzen eher als problematisch einzustufen. Zum einen sind die Schmerzen häufig mit keiner aktiven Entzündung verbunden, zum anderen ist das schleimhautschädigende Potenzial von nichtsteroidalen Antirheumatika auf den oberen und unteren Gastroinstinaltrakt nicht zu unterschätzen. Opiate stellen nicht zuletzt durch ihre Aktivierung analgetisch wirkender deszendierender Nervenbahnen einen attraktiven Therapieansatz abdominaler Schmerzen dar. Einschränkend muss jedoch hierbei auf die kontraktionsfördernde Wirkung opioiderger Substanzen hingewiesen werden. Hieraus resultiert eine Limitierung des Einsatzspektrums einer Vielzahl der aktuell für den klinischen Einsatz verfügbaren Opiaten. Von trizyklischen Antidepressiva ist bekannt, dass sie in bereits geringen Dosierungen ihre analgetische Wirkung entfalten können. Neue Wege der Analgesie werden aktuell durch die Entwicklung und den Einsatz von Substanzen beschritten, welche die serotoninergen Bahnen beeinflussen.

Der in der Modulation gastrointestinaler Motilität eingesetzte Wirkstoff Dompe- ridon erhöht interessanterweise viszerale Wahrnehmungsschwellen. Bei Vorliegen persistierender krampfartiger Abdominalschmerzen bietet sich ein Therapieversuch mit Mebeverin oder aber auch mit Pfefferminzöl an. Vor allem bei dyspeptischen Beschwerden vom sog. Ulkustyp ist der Einsatz von Protonenpumpeninhibitoren durch eine Hemmung der Magensäure ein „First-line-Medikament“. Die H.-pylori-Eradikationstherapie dagegen ist umstritten und scheint nur bei etwa $2-10 \%$ der Patienten mit funktioneller Dyspepsie und $H$. pylori effektiv zu sein. Wahrscheinlich sind selbst diese begrenzten „Erfolge“ zumindest bei einem Teil der Patienten darauf zurückzuführen, dass ein peptisches Ulkus nicht diagnostiziert werden konnte. Ein interessanter Behandlungsansatz sind schließlich psychologische Therapien, teils in Verbindung mit medikamentösen Therapien, aber auch alternative Therapien, so z. B. mit Phytotherapeutika.

\section{Fazit}

Das Symptom „Bauchschmerz" stellt oft eine Herausforderung für den damit konfrontierten Arzt dar. Auf der Grundlage von Anamnese, körperlicher Untersuchung und einer Basisdiagnostik muss er rasch über die Ursache des Symptoms und die Dringlichkeit weiterer diagnostischer und therapeutischer Maßnahmen entscheiden. Dazu ist die Unterscheidung zwischen akuten und chronischen Schmerzen wichtig. Ziel ist eine möglichst kausale Therapie des Abdominalschmerzes. Eine symptomatische Analgesie dagegen sollte in der Regel nur transienter Natur sein. Eine Ausnahme bilden hierbei Krankheitsbilder mit unklarer Ätiologie oder fehlenden kausalen Behandlungsoptionen. Während dem akuten Bauchschmerz meist klar definierte strukturelle Läsionen zugrunde liegen, die gezielt angegangen werden können, sind beim chronischen oder chronisch rezidivierenden Schmerz strukturelle Läsionen mit den in der klinischen Routine verfügbaren Techniken oft nicht nachweisbar (• Abb. 6). Es ist jedoch davon auszugehen, dass Fortschritte in der Grundlagenforschung in nächster Zeit auch kausale Behandlungsmöglichkeiten für diese Patienten mit sich bringen.

\section{Korrespondierender Autor} PD Dr. J. M. Gschossmann

Klinik für Gastroenterologie, Inselspital/Universität Bern, CH-3010 Bern E-Mail: juergen.gschossmann@insel.ch

Interessenkonflikt: Der korrespondierende Autor versichert, dass keine Verbindungen mit einer Firma, deren Produkt in dem Artikel genannt ist, oder einer Firma, die ein Konkurrenzprodukt vertreibt, bestehen.

\section{Literatur}

1. Agréus L, Svärdsudd K, Nyrén O, Tibblin G (1995) Irritable bowel syndrome and dyspepsia in the general population: overlap and lack of stability over time. Gastroenterology 109: 671-680

2. Bohner $H$, Yang $Q$, Franke C, Verreet PR, Ohmann C (1998) Simple data from history and physical examination help to exclude bowel obstruction and to avoid radiographic studies in patients with acute abdominal pain. Eur J Surg 164: 777-784

3. Cervero F (1988) Neurophysiology of gastrointestinal pain. Baillieres Clin Gastroenterol 2: 183-199

4. de Dombal FT (1994) Acute abdominal pain in the elderly. J Clin Gastroenterol 19: 331-335

5. Drossman DA, Li Z, Andruzzi E, Temple RD et al. (1993) U.S. Householder survey of functional gastrointestinal disorders. Prevalence, sociodemography, and health impact. Dig Dis Sci 38: 1569-1580

6. Gschossmann JM, Adam B, Liebregts T, Buenger $L$, Ruwe M, Gerken G, Mayer EA, Holtmann G (2002) Effect of transient chemically induced colitis on the visceromotor response to mechanical colorectal distension. Eur J Gastroenterol Hepatol 14: 1067-1072

7. Gschossmann JM, Hauser W, Wesselmann U, Holtmann G (2002) Diagnostic criteria and therapeutic strategies of abdominal pain syndromes. Schmerz 16: 476-480

8. Gschossmann JM, Holtmann G, Mayer EA (2002) Epidemiology and clinical phenomenology of visceral pain. Schmerz 16: 447-451

9. Gschossmann JM, Liebregts T, Adam B, Buenger L, Ruwe M, Gerken G, Holtmann G (2004) Long-term effects of transient chemically induced colitis on the visceromotor response to mechanical colorectal distension. Dig Dis Sci 49: 96-101

10. Heading RC (1999) Prevalence of upper gastrointestinal symptoms in the general population: a systematic review. Scand J Gastroenterol [Suppl] 231: 3-8

11. Holtmann G (2004) IBS: a syndrome or many diseases? Best Pract Res Clin Gastroenterol 18 [Suppl]: 91-97

12. Holtmann G, Goebell H, Talley NJ (1997) Functional dyspepsia and irritable bowel syndrome: is there a common pathophysiological basis? Am J Gastroenterol 92: 954-959

13. Holtmann G, Gschossmann J, Buenger L, Gerken G, Talley NJ (2002) Do changes in visceral sensory function determine the development of dyspepsia during treatment with aspirin? Gastroenterology 123: $1451-1458$

14. Holtmann G, Siffert W, Haag S, Mueller N, Langkafel M, Senf W, Zotz R, Talley NJ (2004) G-protein beta 3 subunit 825 CC genotype is associated with unexplained (functional) dyspepsia. Gastroenterology 126: 971-979 
15. Hyams JS, Burke G, Davis PM, Rzepski B, Andrulonis PA (1996) Abdominal pain and irritable bowel syndrome in adolescents: a community-based study. J Pediatr 129: 220-226

16. Joshi SK, Gebhart GF (2000) Visceral pain. Curr Rev Pain 4: 499-506

17. Jung PJ, Merrell RC (1988) Acute abdomen. Gastroenterol Clin North Am 17: 227-244

18. Lewin GR, Moshourab R (2004) Mechanosensation and pain. J Neurobiol 61:30-44

19. Mayer EA, Gebhart GF (1994) Basic and clinical aspects of visceral hyperalgesia. Gastroenterology 107: 271-293

20. McCleskey EW (2003) New player in pain. Nature 424: 729-730

21. Mertz H (2003) Review article: visceral hypersensitivity. Aliment Pharmacol Ther 17: 623-33

22. Olten KW (2002) Diagnosis of irritable bowel syndrome. Gastroenterology 122: 1701-1714

23. Parry SD, Stansfield R, Jelley D, Gregory W, Phillips E, Barton JR, Welfare MR (2003) Does bacterial gastroenteritis predispose people to functional gastrointestinal disorders? A prospective, community-based, case-control study. Am J Gastroenterol 98: 1970-1975

24. Pearigen $P$ (1996) Unusual causes of abdominal pain. Emerg Med Clin North Am 14: 593-613

25. Purcell T (1989) Nonsurgical and extraperitoneal causes of abdominal pain. Emerg Med Clin North Am 7: 721-740

26. Schima W (2001) Diagnostic imaging of the acute abdomen. Wien Med Wochenschr 151: 532-535

27. Stawowy M, Bluhme C, Arendt-Nielsen L, Drewes AM, Funch-Jensen P (2004) Somatosensory changes in the referred pain area in patients with acute cholecystitis before and after treatment with laparoscopic or open cholecystectomy. Scand J Gastroenterol 39: 988-993

28. Talley NJ, Silverstein MD, Agréus L, Nyrén O, Sonnenberg A, Holtmann G (1998) AGA Technical review: evaluation of dyspepsia. Gastroenterology 114: 582-595

29. Thuluvath PJ, Connolly GM, Forbes A, Gazzard BG (1991) Abdominal pain in HIV infection. Q J Med 286: 275-285

30. Yamamoto W, Kono H, Maekawa H, Fukui T (1997) The relationship between abdominal pain regions and specific diseases: An epidemiologic approach to clinical practice. J Epidemiol 7: 27-32

\section{Verjährungsfristen zum Jahresende bei ausstehenden Privathonoraren beachten}

Ärztliche Honorarforderungen unterliegen der regelmäßigen Verjährungsfrist gemäß § 195 BGB einer 3-jährigen Verjährungsfrist. Die Verjährung beginnt mit dem Schluss des Jahres, in dem der Anspruch entstanden bzw. die Rechnung erstellt ist.

\section{Verzug des Patienten}

Durch $\S 286$ BGB ist geregelt, dass der Patient spätestens dann in Verzug gerät, wenn er nicht innerhalb von 30 Tagen nach Fälligkeit und Zugang der Rechnung zahlt. Ab dem Zeitpunkt des Verzugseintritts (30 Tage nach Rechnungsstellung) hat der Arzt zusätzlich einen Anspruch auf Zinsen. Nach § 12 GOÄ wird der Honoraranspruch des Arztes gegenüber dem Privatpatienten erst fällig, wenn der Arzt eine den Vorschriften der GOÄ entsprechende Rechnung erteilt hat. Mit dieser Rechnung hat es der Arzt grundsätzlich in der Hand, durch verspätete Rechnungsstellung den Verjährungsanspruch hinauszuschieben. Aber auch dies geht nur begrenzt, denn nach der Berufsordnung ( $\$ 14$ MUBO) soll der Arzt seine Rechnungen im Allgemeinen mindestens vierteljährlich stellen.

\section{Verjährungsfristen für Honorarrech- nungen aus dem Jahre 2002}

Nach neuem Recht verjähren Honorarforderungen aus Rechnungsstellungen im Jahr 2002 mit Ablauf des 31.12.2005.

\section{Verhinderung der Verjährung}

Der Arzt kann den Eintritt der Verjährung verhindern. Hierfür reicht es aber nicht, dem Patienten eine oder mehrere Mahnungen zu übersenden. Eine Unterbrechung der Verjährung tritt ein, wenn der Patient die Forderung gegenüber dem Arzt anerkannt hat und wenn der Arzt den Honoraranspruch durch Mahnbescheid oder Klage geltend macht. In diesem Fall muss der Mahnbescheid vor Ablauf der Verjährungsfrist dem Patienten zugestellt sein. Es genügt zwar auch, wenn der Mahnbescheid oder die Klage vor dem 31.12. bei Gericht eingegangen ist. Eine Unterbre- chung der Verjährung tritt aber in diesem Fall nur ein, wenn der Mahnbescheid oder die Klage dem Patienten alsbald zugestellt wird (§ 270 Abs. 3 ZPO). Der sorgfältige Arzt wird deshalb spätestens im Laufe der Monate November und Dezember 2005 seine Honorarrechnungen aus dem Jahre 2002 seinem Rechtsanwalt bzw. seiner ärztlichen Verrechnungsstelle zur gerichtlichen Geltendmachung übergeben oder selbst einen Mahnbescheid beantragen bzw. Klage erheben.

\section{Quelle: M. G. Broglie, Rechtsanwalt, Wiesbaden; www.arztrecht.de E-Mail:broglie@arztrecht.de}

